\title{
Increasing the Laser-Induced Damage Threshold of Optical Components by Atmospheric Pressure Plasma Surface Finishing
}

\author{
Christoph Gerhard*, Marco Stappenbeck, and Daniel Tasche \\ University of Applied Sciences and Arts, Faculty of Natural Sciences and Technology, 37085 Göttingen, Germany
}

\begin{abstract}
In this contribution, a plasma-based approach for finishing optics surfaces is introduced. Experiments were performed on classically manufactured zinc crown glass and sapphire. It is shown that the use of direct dielectric barrier discharge plasma at atmospheric pressure allows the removal of surfaceadherent carbonaceous contaminations that were induced by classical manufacturing. Moreover, the use of such plasma leads to a certain decrease in surface roughness. Both effects, surface cleaning and smoothing finally increase the laser-induced damage threshold of optical components.
\end{abstract}

\section{Introduction}

In the last decades, the laser-induced damage threshold (LIDT) of optics surfaces has gained in importance due to the rapidly growing number of laser applications and the accompanying need of laser beam shaping and imaging systems. However, the LIDT of optics surfaces is limited, e.g. due to micro cracks, subsurface damages, hydrated silica gel layers and light-absorbing near-surface contaminants. These effects and phenomena result from classical optics manufacturing via grinding and chemicalmechanical polishing. In order to avoid or heal such defects, quite different techniques and methods - mainly post-processing steps - were developed and investigated in the past. In this contribution, a novel and promising plasma-based approach is presented.

\section{Materials and methods}

In this work, classically lapped and polished plane plates made of zinc crown glass as well as classically ground and super-polished sapphire samples were investigated. The surfaces were finally plasma treated using a direct dielectric barrier discharge (DBD) plasma operated with argon as process gas [1]. The samples were placed within the discharge gap, consequently acting as dielectric barrier and thus as an essential part of the discharge. In doing so, the plasma was ignited directly on the sample surface that was slightly heated up to a temperature of approximately $90^{\circ} \mathrm{C}$ [2].

The LIDT was determined by the diameter regression technique and the detection of the minimum damage fluence, respectively, as reported in more detail in [3] for zinc crown glass and in [4] for sapphire. LIDT measurements were performed prior to and after plasma treatment. Moreover, the impact of the plasma treatment on surface contamination was investigated via X-ray photoelectron spectroscopy (XPS).

\section{Results and discussion}

\subsection{Zinc crown glass}

By the plasma treatment (for 60 seconds), the carbon content on the zinc crown glass surfaces was notably reduced as shown in Figure 1. The presence of carbon can be attributed to carbonaceous contaminations as induced by the manufacturing process, e.g. by wear debris from the lapping agent or the polyurethane polishing pad [3].

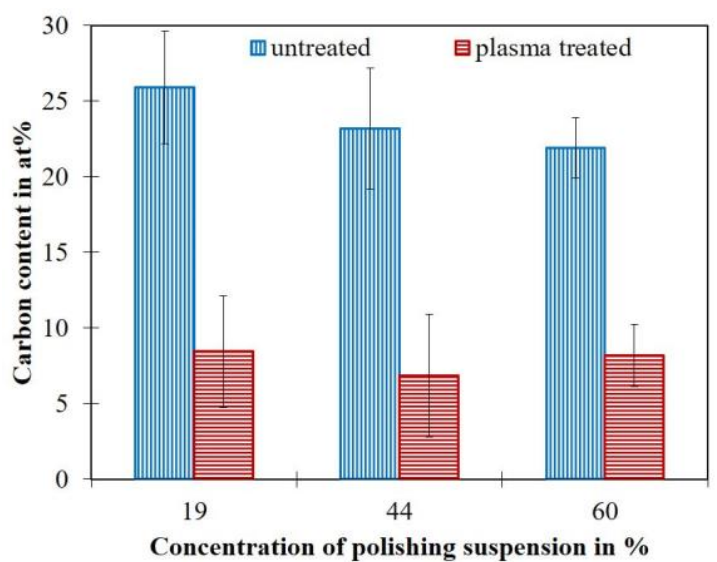

Fig. 1. Carbon content of zinc crown glass surfaces before and after plasma treatment; the samples were polished with different polishing suspension concentrations.

Interestingly, the initial carbon content tends to depend on the concentration of the polishing suspension and the resulting type of interaction between the work piece and the polishing pad surface, respectively. In contrast, the final carbon content after plasma treatment reaches a saturation value. This behaviour can be explained by a plasma-induced removal of carbonaceous contaminants

\footnotetext{
* Corresponding author: christoph.gerhard@hawk.de
} 
from the surface whereas some residues remain in the subsurface region as for example within micro cracks. However, the plasma cleaning leads to an increase in LIDT as shown in Figure 2.

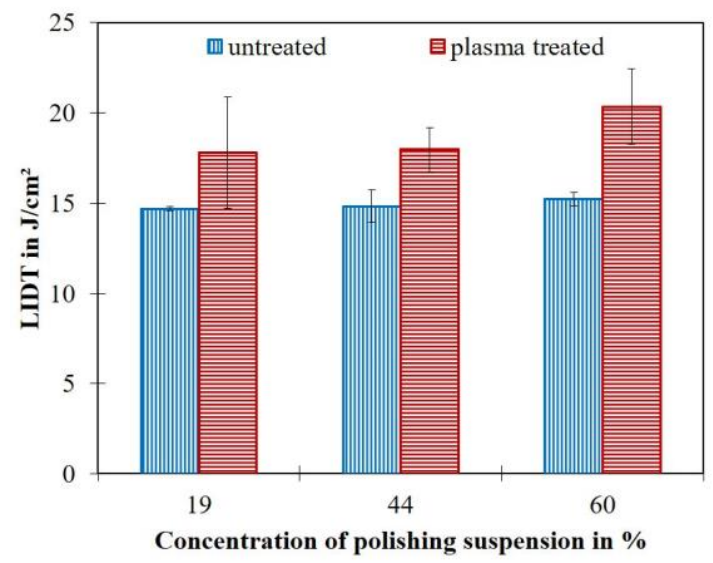

Fig. 2. Laser-induced damage threshold (LIDT) of zinc crown glass surfaces before and after plasma treatment; the samples were polished with different polishing suspension concentrations.

\subsection{Sapphire}

A plasma-induced removal of carbonaceous contaminants from the surface was also observed in the case of superpolished sapphire as shown in Figure 3.

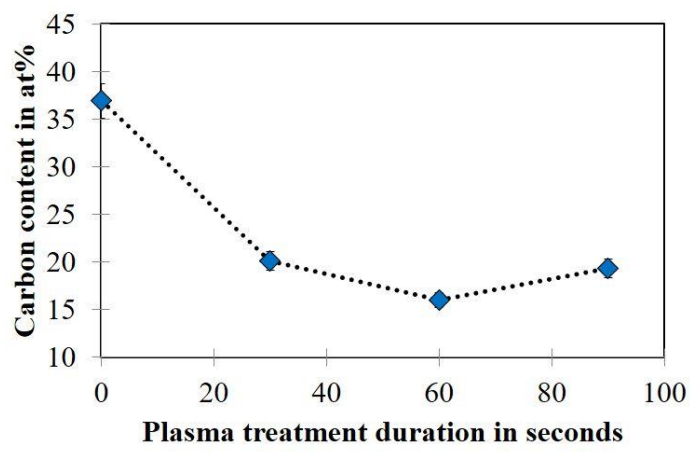

Fig. 3. Carbon content of sapphire surfaces vs. plasma treatment duration.

In addition to XPS measurements, the impact of the plasma treatment on surface roughness was investigated via atomic force microscopy (AFM) measurements where the quadratic mean area roughness $\mathrm{Sq}$ of the sample surfaces before and after plasma treatment was detected. As shown in Figure 4, the plasma treatment effected a certain surface smoothing.

This effect was already observed in previous work $[1,5]$ and leads to a reduction of diffusively scattered light at the surface, consequently contributing to an increase in LIDT. The increase in LIDT of the investigated sapphire surface as shown in Figure 5 can thus be explained by both plasma-induced effects, surface cleaning and smoothing. It finally turns out that after a plasma treatment duration of 90 seconds, the LIDT is increased by a factor of 1.7 .

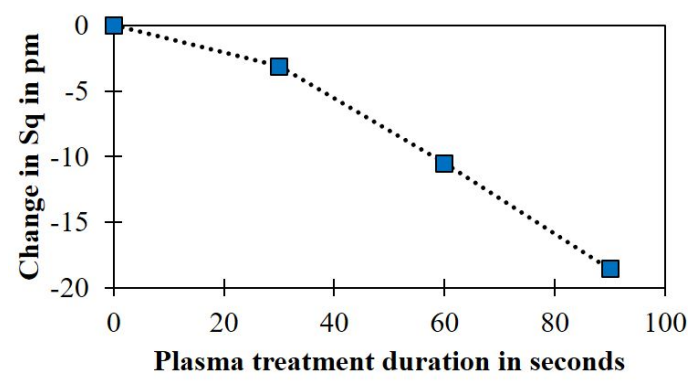

Fig. 4. Change in quadratic mean area roughness $\mathrm{Sq}$ of sapphire surfaces vs. plasma treatment duration.

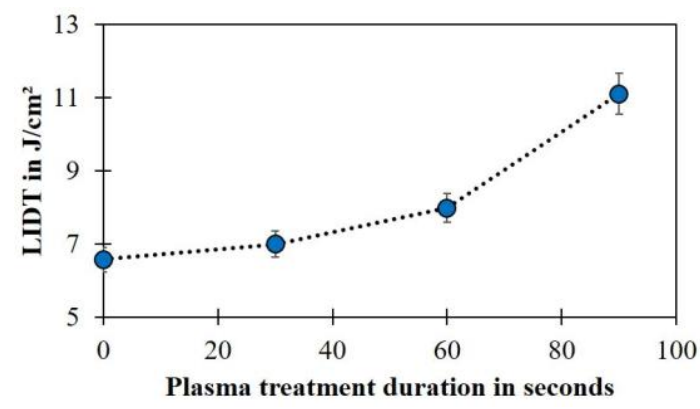

Fig. 5. Laser-induced damage threshold (LIDT) of sapphire surfaces vs. plasma treatment duration.

\section{Conclusions}

The observed increase in LIDT is of great interest for the manufacture of laser optics and protection windows. The application of a direct DBD plasma represents a costefficient approach with a high degree of efficiency. In addition to cleaning, surface smoothing is a further advantageous plasma-induced effect. The short process time makes this technique applicable on industrial scale where the "cold" plasma treatment at temperatures well below the glass softening temperature and the possibility to work at atmospheric pressure are additional benefits.

The authors would like to thank Helene Dyck from IMPEX HighTech GmbH in Rheine as well as Lutz Müller and Robert Köhler from the University of Applied Sciences and Arts in Göttingen for their help during the preparation and analysis of the investigated samples.

\section{References}

1. C. Gerhard, T. Weihs, A. Luca, S. Wieneke, W. Viöl, J. Eur. Opt. Soc.-Rapid 8, 13081 (2013)

2. A. Gredner, C. Gerhard, S. Wieneke, K. Schmidt, W. Viöl, J. Mater. Sci. Eng. B 3, 346 (2013)

3. C. Gerhard, M. Stappenbeck, Appl. Sci. 8, 1556 (2018)

4. C. Gerhard, D. Tasche, N. Munser, H. Dyck, Opt. Lett. 42, 49 (2017)

5. C. Gerhard, S. Roux, S. Brückner, S. Wieneke, W. Viöl, Appl. Opt. 51, 3847 (2012) 